\title{
Was ist neu in der Allergiepråvention?
}

\section{Liebe Leser,}

die weiterhin hohe Prävalenz allergischer Erkrankungen und die eingeschränkten Möglichkeiten einer kausalen Therapie geben der Allergieprävention eine besondere Bedeutung. Während über Jahrzehnte der Gedanke der vorbeugenden Karenz die präventiven Empfehlungen bestimmte, prägt inzwischen die Unterstützung der natürlichen Toleranzentwicklung die empfohlenen Maßnahmen. Diesen Richtungswechsel beschreibt auch die inzwischen mehrfach überarbeitete S3-Leitlinie. In diesem Buch werden, aufbauend auf dem Update 2014 der S3-Leitlinie „Allergieprävention“, die wesentlichen Aspekte hierzu unter Berücksichtigung der aktuellen Literatur besprochen. Freuen Sie sich auf die im Folgenden kurz skizzierten Kapitel:

Einführend wird das Thema aus der Sicht der wesentlich beteiligten medizinischen Fachdisziplinen beleuchtet: Pädiatrie (Lars Lange), Dermatologie (Antonia Schreiber et al.), HNO-Heilkunde (Ingrid Caspari et al.) und Pneumologie (Christian Vogelberg). Nachfolgend führt Harald Renz in die immunologischen Grundlagen der Toleranz ein.

Im Themenbereich Ernährung bespricht zunächst Matthias Kopp das Thema Stillen und stellt die aktuelle Studienlage, aber auch die methodischen Schwierigkeiten dar. Die aktuelle Datenlage unterstützt weiterhin überwiegend die Empfehlung, dass für den Zeitraum der ersten vier Monate voll - im Sinne der WHO-Definition von ,predominant breastfeeding“-gestillt werden soll. Zahlreiche Studien deuten darauf hin, dass eine Beikosteinführung ab Beginn des 5. Lebensmonats mit einer geförderten Toleranzentwicklung assoziiert ist. Entsprechend gibt es Hinweise, dass längeres ausschließliches Stillen auch mit einer Risikoerhöhung für Allergien verbunden sein kann.

Für Kinder mit erhöhtem Allergierisiko, die nicht gestillt werden können, sehen die aktuellen Empfehlungen zur Primärprävention von allergischen Erkrankungen die Gabe von Hydrolysatnahrungen in den ersten vier Lebensmonaten vor. Allerdings wird der protektive Nutzen von partiell bzw. extensiv hydrolysierten Formulanahrungen inzwischen zunehmend kontrovers diskutiert. Birgit Ahrens widmet sich in ihrem Kapitel „Hydrolysatnahrungen“ diesem Thema umfassend, indem sie auf die Unterschiede von Zusammensetzung und Hydrolysegrad von Muttermilchersatznahrungen eingeht und die große Heterogenität der klinischen Studien betrachtet. Sie stellt die aktuellen Anforderungen an die Hersteller durch die EFSA (Delegierten Verordnung (EU) 2016/127) und deren Forderung nach einem produktspezifischen kurz- und langfristigen Wirksamkeitsnachweis anhand von klinischen Studien dar. Ergänzend geht sie auf den Einfluss einer frühkindlichen Formula-Ernährung auf diverse, langanhaltende Gesundheitsaspekte ein.

Einer gesunden und vielseitigen Ernährungsweise als Grundlage einer guten Immunantwort kommt eine entscheidende Rolle im Rahmen allergieverhindernder Maßnahmen zu. Deshalb wurden diesem Aspekt drei eigenständige Kapitel gewid- 
met: Neben den Erkenntnissen zur Ernährung während Schwangerschaft und Stillzeit sowie im Beikostalter wird in einem gesonderten Kapitel auf das Thema Fette eingegangen. In letzterem Kapitel werden vor allem auf die hochungesättigten Omega-3-Fettsäuren besprochen. Aktuelle Studienergebnisse legen nahe, dass die Versorgung der Mutter mit diesen Fettsäuren eine wichtige schützende Rolle hinsichtlich der Entwicklung allergischer Erkrankungen einnimmt.

Während frühere Ansätze vor allem auf die Vermeidung häufiger Auslöser kleinkindlicher Nahrungsmittelallergien abzielten, stehen heute vor allem Vielfalt, Qualität und Integration möglichst aller Lebensmittelgruppen im Vordergrund. Ob häufige Auslöser kleinkindlicher Nahrungsmittelallergien gezielt verzehrt werden sollten, ist nicht abschließend geklärt. Beim mütterlichen Verzehr in Schwangerschaft und Stillzeit spricht die Datenlage insgesamt eher dafür, dass die Exposition das kindliche Immunsystem trainiert. Voraussetzung dafür ist allerdings, dass die Exposition im Mutterleib und durch die Muttermilch auch tatsächlich stattfindet. Bei den Kindern selbst ist eher davon auszugehen, dass die Fülle an Exposition mit Umweltfaktoren insgesamt eine wichtige Rolle spielt, während die gezielte und frühzeitige Einführung potenter Nahrungsmittelallergene bisher nicht empfohlen wird.

Ohne Frage und eng assoziiert mit der Ernährung sind auch das Darmmikrobiom und dessen Metabolite wesentlich an der Entwicklung des Immunsystems beteiligt. Remo Frei gibt in seinem Beitrag zu „Pro- und Präbiotika“ einen umfassenden Überblick darüber, welchen Bakterienstämmen als Probiotika eine wichtige Rolle für die Ausbildung von immunologischer Toleranz zukommt und welche Interaktionen mit der Ernährung bestehen. Im Zusammenhang mit Präbiotika geht er vor allem auf die Bildung von kurzkettigen Fettsäuren und deren gesundheitlicher Bedeutung ein.

Margitta Worm widmet sich dem Thema Vitamin D, einem Nährstoff bzw. Hormon, für das für große Teile der Bevölkerung vor allem in den Wintermonaten ein Mangel beschrieben ist und entsprechend eine Vitamin-D-Supplementation für bestimmte Bevölkerungsgruppen empfohlen wird. Ob auch zur Primärprävention allergischer Erkrankungen eine Supplementation empfehlenswert ist, wird kontrovers diskutiert und lässt sich derzeit aufgrund der heterogenen Datenlage nicht abschließend beurteilen.

Das Thema Kaiserschnittentbindung wird in einem Kapitel von Cathleen MucheBorowski behandelt. Dies trägt der Evidenzlage Rechnung, die ein erhöhtes Risiko insbesondere für Asthma bei Kindern zeigt, die durch Kaiserschnitt auf die Welt kamen. Die mangelnde Immunstimulation durch die Exposition im natürlichen Geburtskanal wird hier u. a. als ursächlich diskutiert. Entsprechend wurden andere immunologische Phänotypen bei diesen Kindern beobachtet. Auch Veränderungen der Lungen- und Leberfunktion sowie des Stressverhaltens wurden bei diesen Kindern beschrieben. Vor dem Hintergrund, dass derzeit in Deutschland rund jedes dritte Kind durch Kaiserschnitt auf die Welt kommt, sollte dieser Umstand bei der Wahl des Geburtsverfahrens berücksichtigt werden. 
Die aktuelle Studienlage zur Haustierhaltung und zur Hausstaubmilbenexposition wird in dem Kapitel von Susanne Lau beleuchtet und bestätigt im Wesentlichen die bisherigen Empfehlungen. Weiterhin werden diesbezüglich keine Einschränkungen für Nicht-Risikokinder empfohlen. Die Ergebnisse für Hunde- und Katzenhaltung sind weiterhin unterschiedlich. Hundehaltung ist nach aktuellen Metaanalysen mit einer Risikoreduktion für das atopische Ekzem und Asthma verbunden. Katzenhaltung geht diesen Metaanalysen zufolge, bei heterogener Einzelstudienlage, nicht mit einem erhöhten oder erniedrigten Risiko für atopische Erkrankungen einher. Allerdings geben Einzelstudien bei Risikokindern, z. B. mit einer Loss-of-function-Mutation im Filaggrin-Gen, ein deutlich erhöhtes Ekzemrisiko bei Katzenhaltung an. Wenig verändert hat sich die Studienlage zur Reduktion des Hausstaubmilbenallergengehalts als primärpräventive Einzelmaßnahme. Ein Cochrane Review aus dem Jahr 2009, der drei interventionelle Kohortenstudien zusammenfasst, zeigt keinen präventiven Effekt. Entsprechend wurden derartige Maßnahmen zur Primärprävention nicht empfohlen. Dies betrifft nicht Maßnahmen zur Sekundär- und Tertiärprävention, wo durchaus Belege der Wirksamkeit existieren.

Auf die Bedeutung der aktiven und passiven Tabakrauchexposition geht Thomas Spindler in seinem Beitrag ein. Unstrittig ist, dass es viele gesundheitliche Gründe gibt, Kinder bereits vor der Geburt vor einer Passivrauchexposition zu schützen, ganz abgesehen von den Folgen des aktiven Rauchens. Wie in dem Beitrag dargelegt, ist allerdings die Studienlage zu klinischen Endpunkten der Atopie bisweilen uneinheitlich. Am stärksten sind die Effekte der Passivrauchexposition offensichtlich auf das Asthma und Asthmasymptome, interessanterweise gibt es auch Hinweise auf Risikoerhöhungen für atopisches Ekzem und Nahrungsmittelallergie. Mit Recht wird in diesem Kapitel auf die methodischen Fallstricke der Ergebnisinterpretation hingewiesen, beispielsweise bei Studien mit vorbelasteten Eltern, in denen selektives Präventionsverhalten zu einer systematischen Verzerrung der Ergebnisse führen kann.

In seinem Beitrag $\mathrm{zu}$ Luftschadstoffen hat Joachim Heinrich die Studienlage insbesondere $\mathrm{zu}$ Feinstaub $\left(\mathrm{PM}_{2,5}\right)$ und Stickstoffdioxid sehr genau untersucht und kommt zu einer ähnlichen Schlussfolgerung wie Thomas Spindler beim Tabakrauch. Während es zahlreiche gute Belege für negative gesundheitliche Auswirkungen der Feinstaub- und Stickoxidexposition gibt, ist die Studienlage zu atopischen Endpunkten widersprüchlich und teilweise von methodischen Einschränkungen begleitet. Am ehesten lassen sich wiederum Effekte auf das Bronchialsystem erkennen. Unstrittig ist auch, dass Exazerbationen bestehender allergischer Erkrankungen durch Luftschadstoffe ausgelöst werden können. Im Bereich der Primärprävention bleibt das Bild allerdings unklar.

Als weitere Schadstoffgruppe werden in dem Kapitel von Hans-Günther Wahl die sogenannten Weichmacher (Phthalate) in ihren Eigenschaften sowie Einsatzgebieten dargestellt und hinsichtlich ihrer gesundheitlichen Gefahren besprochen. Experimentelle wie auch erste epidemiologische Studien weisen darauf hin, dass die Exposition insbesondere mit Asthma und Atemwegssymptomen assoziiert ist, wobei viele der 
epidemiologischen Untersuchungen methodisch angreifbar sind. Die Maßnahmen zur effektiven Reduzierung der Weichmacherbelastung werden besprochen.

Den Einfluss von Medikamenten, insbesondere Schmerzmittel, Antibiotika und Kontrazeptiva, auf atopische Erkrankungen hat Cathleen Muche-Borowski in einem weiteren Kapitel sorgfältig analysiert. Dabei legen zahlreiche Studien Assoziationen zwischen Medikamenteneinnahmen, vor allem von Antibiotika und Paracetamol, und atopischen Erkrankungen, insbesondere Asthma, nahe. Aufgrund potenziell verzerrender Einflussfaktoren (reverse causality) sind diese Ergebnisse vorsichtig zu interpretieren. Subgruppenanalysen von Studien, die diesen Einfluss minimieren konnten, zeigen, dass in diesen Studien keine signifikanten Assoziationen mehr beobachtet wurden.

Die Empfehlung, dass alle Kinder, auch Risikokinder, nach den Empfehlungen der STIKO geimpft werden sollten, ist auch in der aktuellen Leitlinienversion beibehalten worden. Die Gründe für den individuellen und kollektiven Schutz vor Infektionserkrankungen sind offensichtlich und gut belegt. Dass Impfen nicht zu Allergien führt, mitunter sogar vor ihnen schützt, kann in dem Kapitel von Sebastian Schmidt und Tobias Ankermann nachgelesen werden.

Die Bedeutung psychosozialer Einflüsse auf die Allergieentstehung verdeutlicht die wachsende Zahl entsprechender Studien und Beobachtungen. So wurde gezeigt, dass schwerwiegender Lebensereignisse (Trennung der Eltern, Tod eines Elternteils etc.) sowohl in der Schwangerschaft als auch in der frühen Kindheit das Risiko für nachfolgende atopische Erkrankungen erhöht. Ein präventiver Ansatz könnte sich durch die frühzeitige therapeutische Begleitung dieser Kinder ergeben. Der Themenpunkt wird hier nicht in einem gesonderten Kapitel besprochen, ist aber in der Leitlinie (Kapitel 17) enthalten und wird dort diskutiert.

Wir hoffen, dass dieses Buch für Sie eine lohnende und der Allergieprävention dienliche Lektüre darstellt und wünschen Ihnen einen angenehmen Erkenntnisgewinn.

München und Immenstadt, 2019

Imke Reese, Torsten Schäfer 